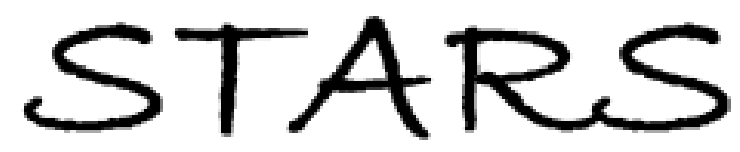

University of Central Florida

STARS

$1-1-2011$

\title{
Precise, real-time, single-shot carrier-envelope phase measurement in the multi-cycle regime
}

\author{
M. Möller \\ University of Central Florida
}

A. M. Sayler

T. Rathje

M. Chini

University of Central Florida

Zenghu Chang

University of Central Florida

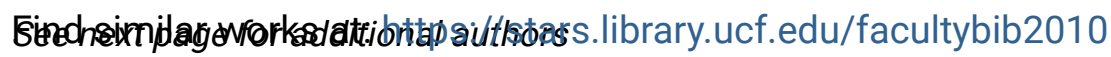

University of Central Florida Libraries http://library.ucf.edu

This Article is brought to you for free and open access by the Faculty Bibliography at STARS. It has been accepted for inclusion in Faculty Bibliography 2010 s by an authorized administrator of STARS. For more information, please contactSTARS@ucf.edu.

\section{Recommended Citation}

Möller, M.; Sayler, A. M.; Rathje, T.; Chini, M.; Chang, Zenghu; and Paulus, G. G., "Precise, real-time, singleshot carrier-envelope phase measurement in the multi-cycle regime" (2011). Faculty Bibliography 2010 s. 1680.

https://stars.library.ucf.edu/facultybib2010/1680

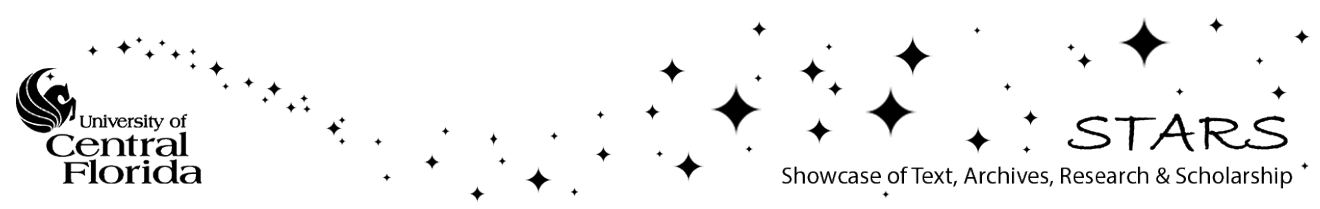


Authors

M. Möller, A. M. Sayler, T. Rathje, M. Chini, Zenghu Chang, and G. G. Paulus 


\section{Precise, real-time, single-shot carrier- envelope phase measurement in the multi- cycle regime}

Cite as: Appl. Phys. Lett. 99, 121108 (2011); https://doi.org/10.1063/1.3641472

Submitted: 18 June 2011. Accepted: 29 August 2011 . Published Online: 20 September 2011

M. Möller, A. M. Sayler, T. Rathje, M. Chini, Zenghu Chang, and G. G. Paulus

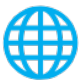

\section{ARTICLES YOU MAY BE INTERESTED IN}

Real-time, single-shot, carrier-envelope-phase measurement of a multi-terawatt laser Applied Physics Letters 110, 081105 (2017); https://doi.org/10.1063/1.4976695

Single-shot velocity-map imaging of attosecond light-field control at kilohertz rate Review of Scientific Instruments 82, 093109 (2011); https://doi.org/10.1063/1.3639333

High harmonic generation in ZnO with a high-power mid-IR OPA

Applied Physics Letters 110, 061101 (2017); https://doi.org/10.1063/1.4975362

\section{Applied Physics Reviews} Now accepting original research 


\title{
Precise, real-time, single-shot carrier-envelope phase measurement in the multi-cycle regime
}

\author{
M. Möller, ${ }^{1,2}$ A. M. Sayler, ${ }^{1,3}$ T. Rathje, ${ }^{1,3}$ M. Chini, ${ }^{2}$ Zenghu Chang, ${ }^{2}$ and G. G. Paulus ${ }^{1,3,4, a)}$ \\ ${ }^{1}$ Institut für Optik und Quantenelektronik, Friedrich-Schiller-Universität Jena, Max-Wien-Platz 1, Jena 07743 , \\ Germany \\ ${ }^{2}$ CREOL and Department of Physics, University of Central Florida, Orlando, Florida 32816, USA \\ ${ }^{3}$ Helmholtz Institut Jena, Max-Wien-Platz 1, Jena 07743, Germany \\ ${ }^{4}$ Department of Physics, Texas A\&M University, College Station, Texas 77843, USA
}

(Received 18 June 2011; accepted 29 August 2011; published online 20 September 2011)

Polarization gating is used to extend a real-time, single-shot, carrier-envelope phase (CEP) measurement, based on high-energy above-threshold ionization in xenon, to the multi-cycle regime. The single-shot CEP precisions achieved are better than 175 and $350 \mathrm{mrad}$ for pulse durations up to $10 \mathrm{fs}$ and $12.5 \mathrm{fs}$, respectively, while only $130 \mu \mathrm{J}$ of pulse energy are required. This opens the door to study and control of CEP-dependent phenomena in ultra-intense laser-matter interaction using optical parametric chirped pulse amplifier based tera- and petawatt class lasers. (ㅇ 2011 American Institute of Physics. [doi:10.1063/1.3641472]

Although a multitude of carrier-envelope phase (CEP) effects in laser-matter interactions produced by few-cycle pulses have been predicted, measured, and utilized, ${ }^{1-3}$ investigation and control of CEP effects with multi-cycle pulses is still a burgeoning field. Of particular interest are phenomena expected for ultra-intense laser-matter interaction driven by short pulse optical-parametric chirped-pulse amplification (OPCPA) based tera- and petawatt class lasers, ${ }^{4}$ such as electron acceleration ${ }^{5}$ and sequential multiple ionization ${ }^{6}$ or applications in the generation of intense isolated attosecond pulses. $^{7}$ Accomplishing these goals requires either control or measurement of the CEP. As typical repetition rates (a few $\mathrm{Hz}$ ) of most tera- and petawatt class lasers exclude implementing standard CEP locking, a precise, real-time, single CEP measurement of every multi-cycle pulse is ideal.

Single-shot CEP measurement of few-cycle pulses based on rescattered high-energy electron spectra using abovethreshold ionization (HATI) in Xe was demonstrated by Wittmann $\mathrm{et} \mathrm{al}^{8}{ }^{8}$ This technique was recently improved to a high precision, single-shot, real-time, carrier-envelope phase meter (CEPM), ${ }^{9,10}$ thereby facilitating CEP tagging, which allows investigation of CEP-dependent effects without the requirement of CEP stabilization. ${ }^{11}$ Alternatively, the measurement can serve as CEP-feedback mechanism to enhance CEP stabilization. ${ }^{12}$ The CEPM utilizes a stereographic measurement of the energy-dependent ATI spectrum to determine left-right asymmetry parameters from two energy regions, which exhibit roughly a sinusoidal CEP dependence shifted by $90^{\circ}$ to each other. ${ }^{9}$ Plotting these two asymmetries against one another, for each laser shot, yields a parametric asymmetry plot (PAP) in which the polar angle, $\vartheta$, corresponds to the CEP, $\varphi$, as $\vartheta \approx \varphi+\varphi_{0}$ and the radius, $r$, can be used to determine the pulse duration of every single lasershot at $\mathrm{kHz}$ repetition rates, ${ }^{13}$ see Fig. 1(a). Here, $\varphi_{0}$ is an arbitrary but fixed offset phase.

The precision of the CEP measurement, $\Delta \phi$, is dominated by statistical error due to electron counting and can be approxi-

\footnotetext{
${ }^{\text {a) }}$ Author to whom correspondence should be addressed. Electronic mail: gerhard.paulus@uni-jena.de.
}

mated as $\Delta \phi \cong \Delta r / r \cdot{ }^{9}$ In our measurement, the radial deviation, $\Delta r$, is typically $\sim 0.1$, independent of the pulse duration. As the radius decreases for multi-cycle pulses, $\Delta \varphi$ increases and single-shot CEP precision is reduced. Therefore, this technique is no longer practical if the pulse duration approaches $8.5 \mathrm{fs}$, where CEP uncertainty exceeds $\sim 350 \mathrm{mrad}\left(\sim 20^{\circ}\right)$.

In this letter, we use polarization gating (PG), a technique well known for its use in generating isolated attosecond pulses from multi-cycle lasers, ${ }^{14,15}$ to achieve high precision single-shot, real-time CEP measurement over an extended range of pulse durations. This facilitates detailed investigation of CEP phenomena in laser matter interaction of few-cycle and multi-cycle lasers, particularly if they are generated with low repetition rates.

In our experimental setup, see Fig. 1(b), the output of the Ti:Sapphire Femtopower ${ }^{\mathrm{TM}}$ Compact $^{\mathrm{TM}}$ Pro HP/HR CEP $(28$

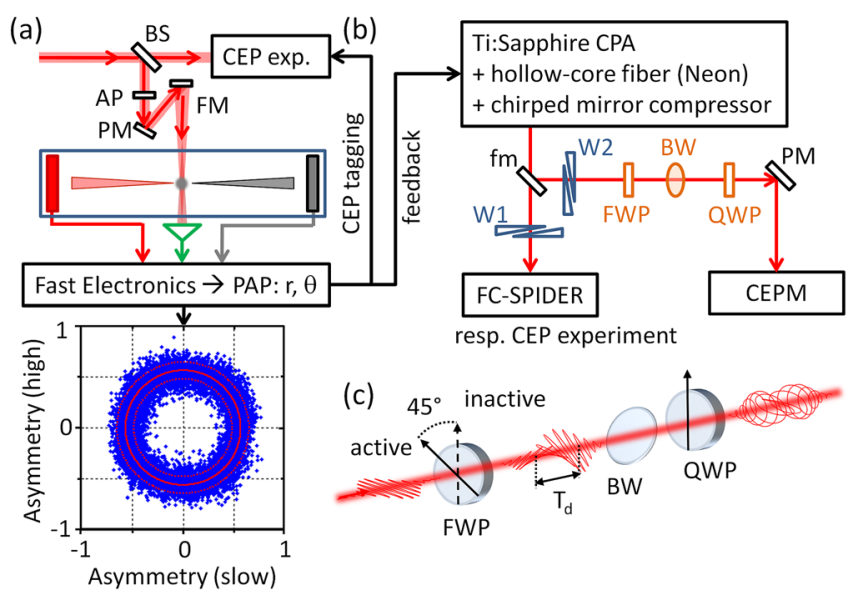

FIG. 1. (Color online) (a) The CEPM delivers coordinates $(r, \vartheta)$ of each laser shot in the PAP within $20 \mu$ s using fast standalone electronics, see Refs. 8 and 9. The beam is only weakly focused (FM) and pulse energy is controlled by an adjustable aperture (AP). (b) Experimental setup to study enhancing effects of polarization gating (PG) as a function of pulse duration. fm - flip mirror, the PG optics FWP - full wave plate, BW - Brewster window, QWP - zero order quarter wave plate, PM - plane mirror. Replacing the fm with a beam splitter and the FC-SPIDER with a primary CEP dependent experiment allows CEP tagging. (c) Birefringent optics used to generate the $\mathrm{PG}$ pulse from in going linear polarization. 
$\mathrm{fs}, \sim 1 \mathrm{~mJ}, 4 \mathrm{kHz}$ ) is spectrally broadened in a neon-filled hollow core fiber and compressed by chirped mirrors and fused silica wedges. The initial pulse duration is changed by controlling the spectral bandwidth using the gas pressure in the hollow core fiber. It is measured with a commercially available SPIDER that is specifically designed for few-cycle pulses (FC SPIDER by APE $\mathrm{GmbH}$ ). ${ }^{16}$ A flip mirror (fm) redirects the beam through birefringent quartz optics to form the PG pulse, while the wedges (W1, W2) are tuned to achieve the shortest pulse in the FC-SPIDER as well as in the CEPM when the PG is inactive, i.e., when the optic axes of all polarizing optics are aligned with the incoming polarization of the pulse (inactive PG). For a CEP tagging measurement, one would replace the flip mirror with a beam splitter and the FC-SPIDER with the CEP experiment of interest.

Our PG optics, Fig. 1(c), consist of a multi-order quartz full-wave plate (FWP) with optic axis $45^{\circ}$ to the incoming linear polarization, a pellicle Brewster window (BW), and a zero-order quarter wave-plate (QWP) with optic axis $0^{\circ}$ to the incoming linear polarization. The duration of the resulting linear PG in the overlap region of the counter rotating elliptical polarized pulses is given by ${ }^{7}$

$$
\delta t_{G}=\frac{\varepsilon \xi_{t h}}{\ln (2)} \frac{\tau^{2}}{T_{d}} .
$$

Here, $\tau$ is the pulse duration and $\varepsilon=0.75$ is the ellipticity outside the PG, which is controlled by the incident angle to the BW. The threshold ellipticity, $\xi_{t h}$, below which the normalized yield of HATI electrons is larger than 0.1 , is $\xi_{t h}=0.15 .{ }^{17}$ The introduced delay time, $T_{d}=n T$, between the counter rotating pulses can be varied in steps of the cycle time, $T=2.7 \mathrm{fs}$, using different thicknesses for the quartz FWP. Deviating angles of the FWP's and the QWP's optic axis to the initial linear polarization are used to fine tune the direction of the linear polarization within the gate and the gate width. ${ }^{18}$

PG restricts the generation of CEP sensitive HATI electrons to a short time window where the laser pulse is linearly polarized by exploiting the quick drop in the yield for increased ellipticity, i.e., in the same way the high harmonic generation is restricted in isolated attosecond pulse generation. This enhances the CEP dependent left-right asymmetries in HATI spectra from multi-cycle laser pulses, thereby improving the CEP sensitivity of the CEPM and extending the upper limit in pulse duration where precise single-shot CEP measurement is accessible. The effectiveness of this approach is shown in Fig. 2(a) where the averaged radius as function of pulse duration with inactive and active PG for three different delays is plotted. Each data point was obtained by averaging the radial coordinate of $\sim 40000$ consecutive laser shots with random CEP after the energy regions were optimized to ensure a linear dependence between $\vartheta$ and the CEP, i.e., a circular distribution in the parametric plot that is identified with a phase shift of $90^{\circ}$ between the two asymmetries. ${ }^{9}$ The single-shot CEP error is shown in Fig. 2(b).

To use a CEPM for CEP tagging or as CEP-feedback mechanism, a precision of at least $20^{\circ}$, i.e., the stability typically achieved with CEP locking, should be maintained. For inactive PG, this benchmark is exceeded below $\sim 8.5$ fs. With PG, the benchmark is achieved at $\sim 10.0$ fs and $\sim 12.5$ fs for delays of $T_{d}=10.8 \mathrm{fs}$ and $T_{d}=13.5 \mathrm{fs}$, respectively. (a)

(b)
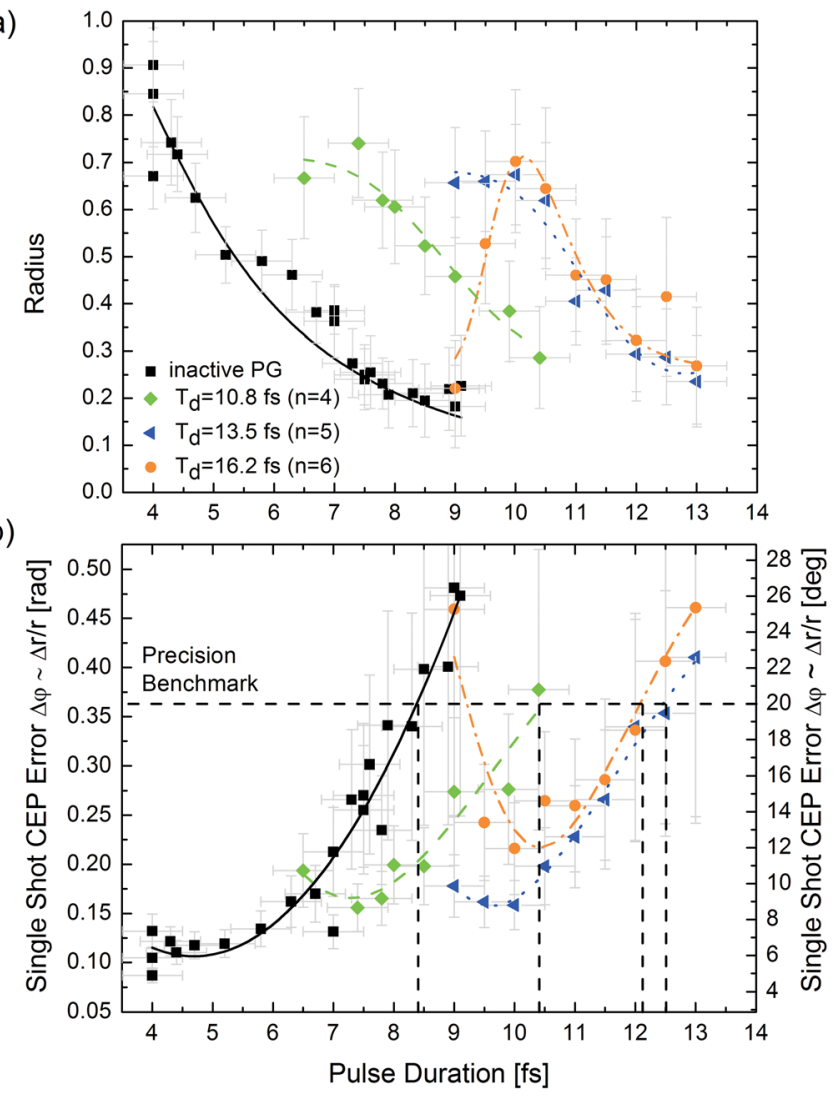

FIG. 2. (Color online) (a) Averaged radius as function of pulse duration with inactive and active PG having retardations. The lines are fitted peak functions to guide the eye for visible convenience. Error bars indicate the standard deviation, $\Delta r$, for radius determination respectively the uncertainty of the FC-SPIDER measurement. (b) Corresponding single-shot CEP error. Horizontal and vertical dashed lines mark when the precision benchmark is exceeded.

Thus, we propose the use of a PG with $T_{d}=10.8$ fs to increase the single-shot CEP precision in the range from 6.5 fs to $8.5 \mathrm{fs}$ and $T_{d}=13.0 \mathrm{fs}$ for pulse durations above $9.0 \mathrm{fs}$. As it can be seen from Eq. (1), the increase in delay is required to maintain a constant gate width of less than or approximately equal to half an optical cycle $\left(\delta t_{G} \cong 1.3 \mathrm{fs}\right)$, which is needed to generate sufficient CEP asymmetries in the left-right HATI spectra. ${ }^{19}$

However, the improvement diminishes when moving to even larger delay as it is shown in the $T_{d}=16.2$ fs data, which has increased CEP-error below 10.0 fs. This behavior can be understood as follows: With respect to the intensity in the elliptically polarized portion of the pulse outside of the gate, a greater delay generates a pulse with a relatively lowered intensity inside the linearly polarized gate (gate intensity). This requires increased pulse energy to keep the gate intensity sufficiently high and in turn enables the direct electrons from outside the gate to reach kinetic energies that are comparable to the CEP sensitive rescattered electrons from inside the gate. These fast direct electrons act as a background signal and cause a lowered signal to noise ratio (SNR) for the CEP measurement which lowers the precision. Additionally, Fig. 2(b) shows that the $T_{d}=16.2$ fs and $T_{d}=13.5$ fs data have a similarly CEP-error above $10.0 \mathrm{fs}$ and it is not possible to push the precision benchmark further using the greater delay. This behavior occurs as longer pulses require longer 
delays to maintain the half cycle gate, what results in a longer leading elliptical part of the pulse outside the gate and simultaneously requires higher pulse energy to keep sufficient gate intensity. The longer the leading edge becomes and the more intensity it has, the more fast direct electrons there will be and the more the target will be depleted at the time of the gate. This mechanism causes an overall upper limit for the applicability of PG to extend real-time, single-shot CEP measurement to the multi-cycle regime.

If one assumes a Gaussian focus and obtains the pulse duration from the PG pulse's envelope numerically, the optimal gate intensity is found to be between $5 \times 10^{13} \mathrm{~W} / \mathrm{cm}^{2}$ and $12 \times 10^{13} \mathrm{~W} / \mathrm{cm}^{2}$ for our measurements. These numbers are consistent with observed cut-off positions in the electron spectra assuming that rescattered electrons generated from the gate follow the well-known cut-off law for linear polarization. ${ }^{20}$ To reach this intensity with linearly polarized 6 fs pulses, 30 $\mu \mathrm{J}$ pulse energy are sufficient while PG pulses with duration of $11 \mathrm{fs}$ and a delay of $T_{d}=13.5$ fs require about four times this pulse energy. Although this is still only a tiny fraction of the available pulse energy at tera- or petawatt laser systems, it will be a limiting factor for lower energy tabletop systems.

Knowing the gate intensity and the required gate width, one can estimate at which pulse duration the target depletion precludes an accurate CEP measurement. The ionization probability as function of pulse duration for three different intensities was calculated using the Ammosov-Delone-Krainov ionization rate, ${ }^{21}$ see Fig. 3. At a pulse duration of $13.0 \mathrm{fs}$, even for the lowest considered gate intensity, $5 \times 10^{13} \mathrm{~W} /$ $\mathrm{cm}^{2}$, only $\sim 10 \%$ of the atoms are left in the ground state and are available to generate CEP sensitive HATI electrons, while $\sim 90 \%$ of the freed electrons are weakly CEP sensitive direct electrons from the elliptical polarized leading edge. This causes lowered SNR and leads to increased single-shot CEP error. Thus, ground state depletion and background signal due to fast direct electrons prevent the applicability of PG at the CEPM to push the limit in pulse duration beyond $12.5 \mathrm{fs}$.

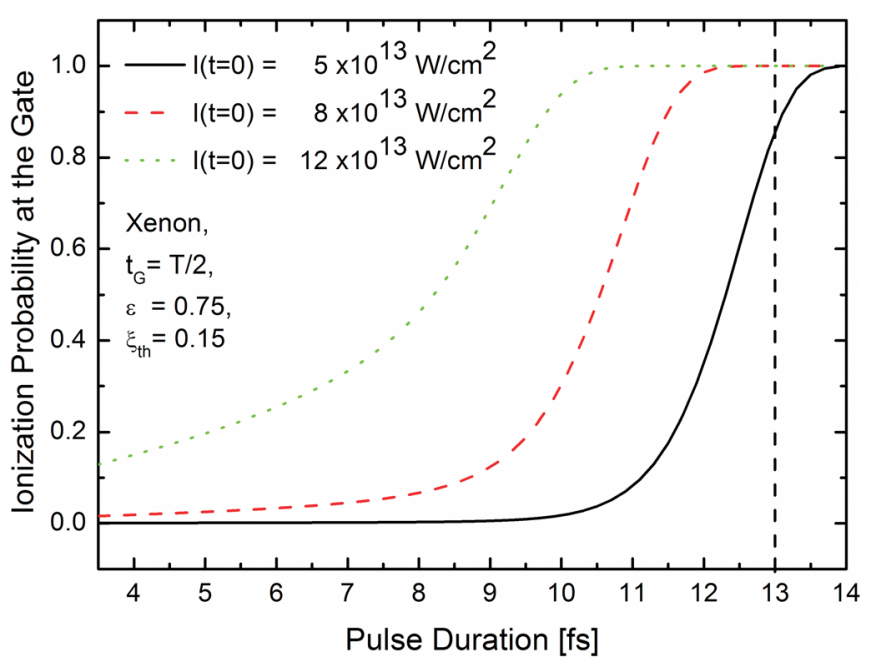

FIG. 3. (Color online) Total ionization probability for the ground state of Xenon gas at the time point of the gate $(t=0)$ calculated for three different gate intensities used in the experiments, $5 \times 10^{13} \mathrm{~W} / \mathrm{cm}^{2}, 8 \times 10^{13} \mathrm{~W} / \mathrm{cm}^{2}$, and $12 \times 10^{13} \mathrm{~W} / \mathrm{cm}^{2}$. The gate width was kept equal to half an optical cycle $\left(t_{G}=1.3 \mathrm{fs}\right)$. The probability is given as $P=1-\exp \left(\int_{-\infty}^{0} w(t) d t\right)$, where $w(t)$ is the Ammosov-Delone-Krainov ionization rate, see Ref. 21.
In conclusion, we extended a precise, real-time singleshot CEP measurement to multi-cycle laser pulses using PG while maintaining all advantages of the technique, ${ }^{9,10}$ e.g., consistent long-term operation and real-time CEP diagnostic within $20 \mu$ s after the laser shot. The technique ${ }^{22}$ achieves single-shot CEP precision below $175 \mathrm{mrad}\left(\sim 10^{\circ}\right)$ for pulse durations up to $10.0 \mathrm{fs}$ and is practical up to $12.5 \mathrm{fs}$ where a precision of $350 \mathrm{mrad}\left(\sim 20^{\circ}\right)$ is reached. The pulse energy consumption is only $\leq 130 \mu \mathrm{J}$ which allows simple data tagging ${ }^{11}$ thereby enabling the study and control of CEP dependent effects, e.g., in ultra-intense laser-matter interaction with OPCPA based tera- and petawatt class lasers. Further, it may serve as a feedback mechanism if CEP stabilization is desired and feasible or as CEP pulse picking criterion for connected experiments.

This work was supported by LaserLab Europe, a Grant PA 730/4 from the German Research Foundation (DFG), and the U. S. Army Research Office under Grant No. W911NF07-1-0475. M. Möller is grateful for being supported by German Academic Exchange Service.

${ }^{1}$ T. Brabec and F. Krausz, Rev. Mod. Phys. 72(2), 545 (2000).

${ }^{2}$ G. G. Paulus, F. Grasbon, H. Walther, F. Villoresi, M. Nisoli, S. Stagira, E. Priori, and S. De Silvestri, Nature 414, 182 (2001).

${ }^{3}$ A. Baltuska, T. Udem, M. Uiberacker, M. Hentschel, E. Goulielmakis, C. Gohle, R. Holzwarth, V. S. Yakoviev, A. Scrinzi, T. W. Hänsch, and F. Krausz, Nature 421, 611 (2003).

${ }^{4}$ D. Herrmann, L. Veisz, R. Tautz, F. Tavella, K. Schmid, V. Pervak, and F. Krausz, Opt. Lett. 34, 2459 (2009).

${ }^{5}$ K. Schmid, L. Veisz, F. Tavella, S. Benavides, R. Tautz, D. Herrmann, A. Buck, B. Hidding, A. Marcinkevicius, U. Schramm, M. Geissler, J. Meyer-ter-Vehn, D. Habs, and F. Krausz, Phys. Rev. Lett. 102, 124801 (2009).

${ }^{6}$ N. I. Shvetsov-Shilovski, A. M. Sayler, T. Rathje, and G. G. Paulus, Phys. Rev. A 83, 033401 (2011).

${ }^{7}$ S. Gilbertson, S. D. Khan, Y. Wu, M. Chini, and Z. Chang, Phys. Rev. Lett. 105, 093902 (2010), and references therein.

${ }^{8}$ T. Wittmann, B. Horvath, W. Helml, M. G. Schatzel, X. Gu, A. L. Cavalieri, G. G. Paulus, and R. Kienberger, Nature Phys. 5, 357 (2009).

${ }^{9}$ A. M. Sayler, T. Rathje, W. Müller, K. Rühle, R. Kienberger, and G. G. Paulus, Opt. Lett. 36, 1 (2011).

${ }^{10}$ G. G. Paulus, K. Rühle, A. M. Sayler, T. Rathje, and W. Müller, German patent pending 10-2010-019-814.5 (5 May 2010).

${ }^{11}$ N. G. Johnson, O. Herrwerth, A. Wirth, S. De, I. Ben-Itzhak, M. Lezius, B. Bergues, M. F. Kling, A. Senftleben, C. D. Schroter, R. Moshammer,J. Ullrich, K. J. Betsch, R. R. Jones, A. M. Sayler, T. Rathje, K. Ruhle, W. Muller, and G. G. Paulus, Phys. Rev. A 83, 013412 (2011).

${ }^{12}$ D. Adolph, A. M. Sayler, T. Rathje, K. Rühle, and G. G. Paulus, "Improved carrier-envelope phase locking of intense few-cycle laser pulses using above-threshold ionization," Opt. Lett. (to be published).

${ }^{13}$ A. M. Sayler, T. Rathje, W. Müller, Ch. Kürbis, K. Rühle, G. Stibenz, and G. G. Paulus, Opt. Express 19, 4464 (2011).

${ }^{14}$ Z. Chang, Phys. Rev. A 70, 043802 (2004).

${ }^{15}$ G. Sansone, E. Benedetti, F. Calegari, C. Vozzi, L. Avaldi, R Flammini, L. Poletto, P. Villoresi, C. Altucci, R. Velotta, S. Stagira, S. De Silvestri, and M. Nisoli, Science 314, 443 (2006).

${ }^{16}$ G. Stibenz and G. Steinmeyer, Rev. Sci. Instrum. 77, 073105 (2006).

${ }^{17}$ G. G. Paulus, F. Zacher, H. Walther, A. Lohr, W. Becker, and M. Kleber, Phys. Rev. Lett. 80, 484 (1998).

${ }^{18}$ C. Marceau, G. Gingras, and B. Witzel, Opt. Express 19, 3576 (2011).

${ }^{19}$ Remark: The result that a gate width of half an optical cycle or less is required for sufficient $\mathrm{CEP}$ asymmetries agrees with the requirement for isolated attosecond pulse generation.

${ }^{20}$ W. Becker, F. Grasbon, R. Kopold, D. B. Milosevic, G. G. Paulus, and H. Walther, Adv. At., Mol., Opt. Phys. 48, 35 (2002).

${ }^{21}$ M. V. Ammosov, N. B. Delone, and V. P. Krainov, Sov. Phys. JETP 64, 1191 (1986).

${ }^{22}$ G. G. Paulus, A. M. Sayler, T. Rathje, M. Möller, and D. Hoff, German patent pending 10-2011-016-441.3 (6 April 6 2011). 